RASĀYAN J. Chem.

Vol. 13 | No. 4 |2515-2522| October - December | 2020 ISSN: 0974-1496 | e-ISSN: 0976-0083 | CODEN: RJCABP

RJC http://www.rasayanjournal.com http://www.rasayanjournal.co.in

\title{
A CORRELATION STUDY OF GROSS CALORIFIC VALUE WITH PARAMETERS OF PROXIMATE ANALYSIS OF COAL
}

\author{
H. Akbar*, Sumath Kumar Laha and Rakesh Saini \\ Chemical Division,National Test House (NWR), Department of Consumer Affairs, Ministry of \\ Consumer Affairs, Food and Public Distribution \\ Government of India,V. K. I. Area, Jaipur-302013, Rajasthan, India \\ *E-mail: akbarchemistry@yahoo.com
}

\begin{abstract}
A correlation study of gross calorific value of coal with parameters of proximate analysis (the content of ash, moisture, volatile matter and fixed carbon) was studied on more than 200 samples of coal from different mining areas across India. Gross calorific value is increased with the decrease of the content of ash, moisture respectively, and gross calorific value is also increased with the increase in the content of volatile matter and fixed carbon respectively. The relevant experimental data and graphs are very useful for industries to estimate the quality of coal. Keywords: Moisture, Ash, Volatile Matter, Fixed carbon, Gross Calorific Value
\end{abstract}

(C) RASĀYAN. All rights reserved

\section{INTRODUCTION}

Coal is one of the best non-renewable energy sources and abundantly available in India and used as the main fuel for the industrial process as well as household use in some parts of the country. India is the second-largest producer and importer of coal in the world. ${ }^{3}$ India's electricity generation highly depends on coal only. ${ }^{3}$ It is used as fuel for power generation for thermal power plants, steel making industries, and cement manufacturing industries, etc., Every country are having coal with different physical/chemical properties. The ash of coal is one of the pozzolanic material used in the manufacturing of cement. Indian coal is having a variety of grades which is dependent on the gross calorific value. Chemical analysis of coal is very important concerning the correlation of gross calorific value with the parameters of proximate analysis which are very useful for the industries to estimate the quality of coal.

\section{Sampling}

\section{EXPERIMENTAL}

Coal samples were pulverized and passed through a sieve 212 micron $^{1}$ as per Indian standard.Estimation of moisture, ash, volatile matter, and fixed carbon was analyzed by Eltra thermogravimetric analyzer (TGA) model Thermostep and results are recorded. Initially we are loading the sample in Eltra thermogravimetric analyzer, within 1.5 hours, we got the results of moisture, ash, volatile matter and fixed carbon respectively.

\section{Gross Calorific Value (GCV)}

Gross calorific value was determined by using a digital bomb calorimeter and results are recorded. ${ }^{2}$ According to environmental condition, we are going to find out water equivalent of a standard benzoic acid, based on that one, find out the calorific value of certified reference material. The calorific value of standard reference material is used for calibration of the digital bomb calorimeter. Then we are going for sample analysis.More than 200 Samples were analyzed and data recorded as follows in Table-1.

\begin{tabular}{c|c|c|c|c|c}
\multicolumn{6}{c}{ Table-1 } \\
\hline S. No. & $\begin{array}{c}\text { Moisture } \\
\% \text { by mass }\end{array}$ & $\begin{array}{c}\text { Ash\% by } \\
\text { mass }\end{array}$ & $\begin{array}{c}\text { GCV, } \\
\text { Kcal/kg }\end{array}$ & $\begin{array}{c}\text { Volatile matter } \\
\% \text { by mass }\end{array}$ & $\begin{array}{c}\text { Fixed Carbon } \\
\% \text { by mass }\end{array}$ \\
\hline 1 & 5.87 & 13.14 & 6300 & 33.22 & 47.77 \\
\hline
\end{tabular}

Rasayan J. Chem., 13(4), 2515-2522(2020)

http://dx.doi.org/10.31788/ RJC.2020.1345842 
RASĀYAN J. Chem.

Vol. 13 | No. 4 |2515-2522| October - December | 2020

\begin{tabular}{|c|c|c|c|c|c|}
\hline 2 & 6.00 & 13.06 & 6270 & 33.11 & 47.83 \\
\hline 3 & 6.09 & 11.92 & 6370 & 34.46 & 47.53 \\
\hline 4 & 6.11 & 11.89 & 6380 & 34.47 & 47.53 \\
\hline 5 & 5.56 & 13.41 & 6280 & 34.12 & 46.92 \\
\hline 6 & 5.57 & 13.44 & 6260 & 33.84 & 47.15 \\
\hline 7 & 5.56 & 13.11 & 6270 & 33.83 & 47.50 \\
\hline 8 & 5.50 & 13.36 & 6250 & 33.53 & 47.61 \\
\hline 9 & 5.49 & 13.36 & 6280 & 33.94 & 47.20 \\
\hline 10 & 5.61 & 13.45 & 6300 & 34.01 & 46.93 \\
\hline 11 & 5.72 & 13.14 & 6240 & 33.38 & 47.76 \\
\hline 12 & 5.59 & 13.34 & 6230 & 33.42 & 47.65 \\
\hline 13 & 6.09 & 13.20 & 6260 & 33.88 & 46.83 \\
\hline 14 & 5.74 & 13.46 & 6250 & 33.51 & 47.28 \\
\hline 15 & 5.65 & 13.46 & 6300 & 33.68 & 47.20 \\
\hline 16 & 5.89 & 13.06 & 6340 & 33.22 & 47.83 \\
\hline 17 & 5.70 & 13.42 & 6330 & 33.51 & 47.37 \\
\hline 18 & 5.88 & 13.13 & 6210 & 33.30 & 47.69 \\
\hline 19 & 5.99 & 13.03 & 6310 & 33.36 & 47.62 \\
\hline 20 & 5.79 & 20.33 & 5730 & 31.80 & 42.08 \\
\hline 21 & 5.77 & 13.45 & 6260 & 34.69 & 46.09 \\
\hline 22 & 5.94 & 12.98 & 6380 & 34.51 & 46.57 \\
\hline 23 & 6.23 & 13.10 & 6240 & 34.61 & 46.06 \\
\hline 24 & 5.99 & 13.00 & 6270 & 34.32 & 46.69 \\
\hline 25 & 5.96 & 13.65 & 6270 & 33.98 & 46.41 \\
\hline 26 & 6.92 & 11.80 & 6350 & 34.63 & 46.65 \\
\hline 27 & 6.73 & 11.88 & 6360 & 34.60 & 46.78 \\
\hline 28 & 6.64 & 11.91 & 6310 & 34.73 & 46.72 \\
\hline 29 & 0.75 & 12.82 & 6210 & 36.67 & 49.77 \\
\hline 30 & 6.08 & 13.21 & 6390 & 34.33 & 46.37 \\
\hline 31 & 5.90 & 13.09 & 6400 & 34.60 & 46.42 \\
\hline 32 & 5.98 & 13.46 & 6190 & 34.57 & 45.98 \\
\hline 33 & 5.90 & 13.10 & 6310 & 33.49 & 47.51 \\
\hline 34 & 6.01 & 13.37 & 6300 & 33.83 & 46.79 \\
\hline 35 & 5.97 & 13.14 & 6300 & 33.67 & 47.22 \\
\hline 36 & 6.25 & 22.05 & 5560 & 30.19 & 41.52 \\
\hline 37 & 5.67 & 13.37 & 6240 & 34.43 & 46.53 \\
\hline 38 & 6.42 & 11.80 & 6360 & 35.23 & 46.55 \\
\hline 39 & 5.77 & 11.77 & 6380 & 36.60 & 45.86 \\
\hline 40 & 4.97 & 13.47 & 6350 & 35.61 & 45.95 \\
\hline 41 & 5.82 & 12.72 & 6280 & 36.51 & 44.95 \\
\hline 42 & 4.96 & 14.42 & 6060 & 34.03 & 46.58 \\
\hline 43 & 5.05 & 13.49 & 6280 & 34.50 & 46.95 \\
\hline 44 & 5.49 & 13.60 & 6040 & 35.47 & 45.44 \\
\hline
\end{tabular}


RASĀYAN J. Chem.

Vol. 13 | No. 4 |2515-2522| October - December | 2020

\begin{tabular}{|c|c|c|c|c|c|}
\hline 45 & 5.38 & 13.61 & 6270 & 35.45 & 45.56 \\
\hline 46 & 5.19 & 13.47 & 6260 & 34.80 & 46.54 \\
\hline 47 & 4.55 & 28.24 & 4800 & 29.18 & 38.03 \\
\hline 48 & 6.27 & 11.82 & 6390 & 35.56 & 46.35 \\
\hline 49 & 6.24 & 15.00 & 5920 & 34.39 & 44.37 \\
\hline 50 & 6.11 & 12.75 & 6140 & 36.28 & 44.86 \\
\hline 51 & 5.66 & 13.58 & 6320 & 34.83 & 45.93 \\
\hline 52 & 6.21 & 12.71 & 6240 & 35.34 & 45.73 \\
\hline 53 & 6.38 & 11.89 & 6350 & 34.99 & 46.74 \\
\hline 54 & 5.61 & 21.28 & 5520 & 33.23 & 40.88 \\
\hline 55 & 5.89 & 13.06 & 6420 & 35.14 & 45.91 \\
\hline 56 & 5.81 & 12.94 & 6420 & 34.32 & 46.93 \\
\hline 57 & 5.63 & 12.10 & 6350 & 36.50 & 45.77 \\
\hline 58 & 6.08 & 12.79 & 6360 & 34.18 & 46.95 \\
\hline 59 & 6.11 & 12.78 & 6210 & 34.08 & 47.04 \\
\hline 60 & 5.97 & 14.41 & 6050 & 33.88 & 45.74 \\
\hline 61 & 6.21 & 12.74 & 6360 & 33.87 & 47.18 \\
\hline 62 & 6.30 & 12.76 & 6260 & 33.89 & 47.05 \\
\hline 63 & 6.21 & 12.75 & 6300 & 33.89 & 47.15 \\
\hline 64 & 6.27 & 12.74 & 6320 & 33.93 & 47.06 \\
\hline 65 & 6.19 & 12.82 & 6290 & 34.03 & 46.96 \\
\hline 66 & 6.16 & 14.38 & 6040 & 33.50 & 45.96 \\
\hline 67 & 6.01 & 11.15 & 6290 & 34.37 & 48.47 \\
\hline 68 & 6.56 & 12.76 & 6190 & 33.53 & 47.15 \\
\hline 69 & 6.51 & 12.75 & 6360 & 33.73 & 47.01 \\
\hline 70 & 6.60 & 12.77 & 5950 & 33.68 & 46.95 \\
\hline 71 & 6.60 & 12.71 & 6180 & 33.53 & 47.12 \\
\hline 72 & 6.66 & 12.80 & 6240 & 33.34 & 47.20 \\
\hline 73 & 6.52 & 12.73 & 6090 & 33.19 & 47.56 \\
\hline 74 & 6.31 & 21.28 & 5830 & 30.44 & 41.97 \\
\hline 75 & 6.74 & 12.74 & 6270 & 33.37 & 47.15 \\
\hline 76 & 5.81 & 11.98 & 5910 & 34.47 & 47.75 \\
\hline 77 & 5.99 & 11.81 & 6040 & 34.07 & 48.13 \\
\hline 78 & 5.94 & 11.81 & 6240 & 34.13 & 48.13 \\
\hline 79 & 5.36 & 13.38 & 6260 & 33.73 & 47.53 \\
\hline 80 & 5.37 & 13.36 & 6120 & 33.64 & 47.63 \\
\hline 81 & 5.46 & 13.40 & 6150 & 33.79 & 47.35 \\
\hline 82 & 5.34 & 13.28 & 6290 & 33.36 & 48.02 \\
\hline 83 & 5.76 & 13.11 & 6210 & 33.73 & 47.40 \\
\hline 84 & 6.44 & 14.00 & 5860 & 32.27 & 47.29 \\
\hline 85 & 5.65 & 13.22 & 6340 & 33.27 & 47.86 \\
\hline
\end{tabular}


RASĀYAN J. Chem.

Vol. 13 | No. 4 |2515-2522| October - December | 2020

\begin{tabular}{|c|c|c|c|c|c|}
\hline 86 & 6.50 & 12.73 & 6270 & 32.91 & 47.85 \\
\hline 87 & 6.03 & 12.29 & 6170 & 33.22 & 48.46 \\
\hline 88 & 6.38 & 11.54 & 6050 & 33.39 & 48.69 \\
\hline 89 & 6.33 & 12.08 & 6240 & 33.65 & 47.93 \\
\hline 90 & 5.88 & 12.42 & 6330 & 32.73 & 48.96 \\
\hline 91 & 5.97 & 13.48 & 5940 & 32.70 & 47.85 \\
\hline 92 & 6.51 & 11.45 & 6080 & 33.25 & 48.79 \\
\hline 93 & 5.89 & 14.44 & 5960 & 32.22 & 47.45 \\
\hline 94 & 6.21 & 12.34 & 6320 & 33.05 & 48.40 \\
\hline 95 & 5.89 & 11.87 & 6390 & 34.18 & 48.07 \\
\hline 96 & 5.76 & 12.98 & 6280 & 33.75 & 47.50 \\
\hline 97 & 5.39 & 12.55 & 6370 & 33.97 & 48.09 \\
\hline 98 & 6.15 & 14.38 & 6000 & 33.43 & 46.04 \\
\hline 99 & 5.91 & 12.06 & 6250 & 35.45 & 46.58 \\
\hline 100 & 6.06 & 12.02 & 6340 & 35.47 & 46.44 \\
\hline 101 & 5.65 & 13.93 & 6070 & 33.93 & 46.49 \\
\hline 102 & 6.08 & 11.42 & 6050 & 34.30 & 48.20 \\
\hline 103 & 5.32 & 14.21 & 6280 & 34.02 & 46.45 \\
\hline 104 & 6.15 & 11.84 & 6210 & 34.99 & 47.02 \\
\hline 105 & 6.27 & 14.49 & 6000 & 33.01 & 46.24 \\
\hline 106 & 6.29 & 11.86 & 6250 & 35.02 & 46.82 \\
\hline 107 & 6.31 & 11.88 & 6040 & 34.70 & 47.10 \\
\hline 108 & 6.26 & 13.34 & 6270 & 33.81 & 46.59 \\
\hline 109 & 6.47 & 11.79 & 6230 & 34.75 & 46.98 \\
\hline 110 & 6.42 & 13.37 & 6340 & 33.63 & 46.58 \\
\hline 111 & 6.55 & 13.32 & 6230 & 33.50 & 46.63 \\
\hline 112 & 6.62 & 11.83 & 6120 & 34.38 & 47.77 \\
\hline 113 & 6.58 & 15.16 & 6050 & 32.72 & 45.54 \\
\hline 114 & 5.65 & 12.78 & 6150 & 35.51 & 46.07 \\
\hline 115 & 5.52 & 12.08 & 6330 & 34.62 & 47.77 \\
\hline 116 & 5.18 & 13.33 & 6380 & 33.46 & 48.04 \\
\hline 117 & 5.21 & 13.02 & 6350 & 33.79 & 47.97 \\
\hline 118 & 5.40 & 13.05 & 6360 & 33.57 & 47.98 \\
\hline 119 & 5.68 & 12.04 & 6390 & 34.77 & 47.51 \\
\hline 120 & 5.60 & 12.34 & 6420 & 33.25 & 48.80 \\
\hline 121 & 5.59 & 13.91 & 6140 & 33.04 & 47.46 \\
\hline 122 & 6.15 & 13.90 & 6010 & 32.91 & 47.05 \\
\hline 123 & 6.30 & 13.48 & 6230 & 32.75 & 47.46 \\
\hline 124 & 5.65 & 12.94 & 6340 & 33.93 & 47.49 \\
\hline 125 & 6.00 & 12.16 & 6300 & 34.32 & 47.53 \\
\hline 126 & 6.08 & 11.47 & 6380 & 34.00 & 48.45 \\
\hline
\end{tabular}


RASĀYAN J. Chem.

Vol. 13 | No. 4 |2515-2522| October - December | 2020

\begin{tabular}{|c|c|c|c|c|c|}
\hline 127 & 5.42 & 13.07 & 6330 & 33.42 & 48.09 \\
\hline 128 & 5.87 & 12.58 & 6380 & 33.29 & 48.26 \\
\hline 129 & 5.49 & 13.37 & 6420 & 33.43 & 47.71 \\
\hline 130 & 5.47 & 13.48 & 6360 & 33.34 & 47.71 \\
\hline 131 & 6.34 & 12.04 & 6380 & 34.09 & 47.53 \\
\hline 132 & 6.25 & 11.98 & 6310 & 33.87 & 47.91 \\
\hline 133 & 6.39 & 11.45 & 6410 & 33.40 & 48.76 \\
\hline 134 & 5.53 & 13.88 & 6390 & 33.67 & 46.92 \\
\hline 135 & 5.98 & 17.15 & 5690 & 33.78 & 43.09 \\
\hline 136 & 6.19 & 12.08 & 6340 & 34.85 & 46.89 \\
\hline 137 & 6.20 & 12.13 & 6320 & 34.59 & 47.08 \\
\hline 138 & 6.28 & 12.07 & 6340 & 35.04 & 46.61 \\
\hline 139 & 6.21 & 12.04 & 6200 & 35.35 & 46.40 \\
\hline 140 & 6.27 & 12.06 & 6120 & 35.20 & 46.47 \\
\hline 141 & 5.58 & 14.11 & 6100 & 33.21 & 47.10 \\
\hline 142 & 6.46 & 13.94 & 6020 & 33.47 & 46.13 \\
\hline 143 & 6.37 & 12.08 & 6300 & 34.65 & 46.89 \\
\hline 144 & 5.59 & 13.59 & 6160 & 34.27 & 46.55 \\
\hline 145 & 6.45 & 11.99 & 6260 & 34.97 & 46.58 \\
\hline 146 & 6.78 & 14.03 & 5870 & 32.73 & 46.47 \\
\hline 147 & 6.54 & 12.04 & 6230 & 34.42 & 47.01 \\
\hline 148 & 6.54 & 12.03 & 6350 & 34.40 & 47.03 \\
\hline 149 & 6.67 & 12.60 & 6140 & 33.52 & 47.21 \\
\hline 150 & 8.57 & 16.15 & 5920 & 31.66 & 43.62 \\
\hline 151 & 5.81 & 13.42 & 6210 & 33.67 & 47.10 \\
\hline 152 & 6.11 & 11.36 & 6180 & 34.22 & 48.31 \\
\hline 153 & 6.86 & 14.42 & 6100 & 33.00 & 45.72 \\
\hline 154 & 5.63 & 13.00 & 6030 & 34.53 & 46.85 \\
\hline 155 & 5.68 & 12.73 & 6300 & 35.34 & 46.24 \\
\hline 156 & 4.82 & 13.55 & 6160 & 33.84 & 47.78 \\
\hline 157 & 5.35 & 13.22 & 6270 & 34.46 & 46.97 \\
\hline 158 & 5.45 & 13.59 & 5870 & 34.97 & 46.00 \\
\hline 159 & 6.06 & 13.94 & 6240 & 33.66 & 46.34 \\
\hline 160 & 5.16 & 18.56 & 6360 & 32.12 & 44.16 \\
\hline 161 & 5.91 & 12.74 & 6150 & 35.48 & 45.86 \\
\hline 162 & 6.24 & 12.09 & 5920 & 34.21 & 47.46 \\
\hline 163 & 5.49 & 13.06 & 6020 & 33.95 & 47.50 \\
\hline 164 & 5.77 & 13.93 & 6180 & 33.51 & 46.79 \\
\hline 165 & 5.83 & 13.00 & 5920 & 34.30 & 46.87 \\
\hline 166 & 5.70 & 13.31 & 6320 & 33.83 & 47.15 \\
\hline 167 & 6.22 & 12.14 & 6310 & 34.98 & 46.65 \\
\hline
\end{tabular}


RASĀYAN J. Chem.

Vol. 13 | No. 4 |2515-2522| October - December | 2020

\begin{tabular}{|c|c|c|c|c|c|}
\hline 168 & 7.45 & 18.58 & 6340 & 30.60 & 43.36 \\
\hline 169 & 6.33 & 14.04 & 6320 & 33.36 & 46.27 \\
\hline 170 & 6.15 & 12.07 & 6150 & 35.24 & 46.54 \\
\hline 171 & 5.87 & 12.49 & 6120 & 33.62 & 48.02 \\
\hline 172 & 6.91 & 15.56 & 5850 & 31.88 & 45.66 \\
\hline 173 & 5.56 & 12.03 & 6080 & 34.45 & 47.96 \\
\hline 174 & 4.91 & 13.59 & 6230 & 34.09 & 47.41 \\
\hline 175 & 5.61 & 12.12 & 6360 & 34.32 & 47.95 \\
\hline 176 & 7.36 & 15.64 & 6200 & 31.76 & 45.23 \\
\hline 177 & 5.89 & 12.06 & 6160 & 34.31 & 47.74 \\
\hline 178 & 4.97 & 13.58 & 6340 & 33.98 & 47.46 \\
\hline 179 & 5.90 & 11.92 & 5840 & 34.16 & 48.02 \\
\hline 180 & 5.89 & 11.97 & 5440 & 34.22 & 47.92 \\
\hline 181 & 5.77 & 13.01 & 5950 & 32.56 & 48.66 \\
\hline 182 & 5.29 & 13.15 & 5700 & 33.01 & 48.55 \\
\hline 183 & 5.88 & 12.13 & 5960 & 33.22 & 48.77 \\
\hline 184 & 5.08 & 13.75 & 6180 & 32.20 & 48.97 \\
\hline 185 & 6.29 & 12.75 & 6280 & 32.97 & 47.98 \\
\hline 186 & 5.65 & 12.98 & 6260 & 32.27 & 49.10 \\
\hline 187 & 6.24 & 14.41 & 5990 & 31.79 & 47.56 \\
\hline 188 & 5.45 & 13.13 & 6210 & 33.02 & 48.40 \\
\hline 189 & 8.86 & 13.39 & 6120 & 31.07 & 46.68 \\
\hline 190 & 6.24 & 12.02 & 6250 & 33.87 & 47.87 \\
\hline 191 & 5.69 & 11.93 & 6150 & 36.17 & 46.21 \\
\hline 192 & 5.61 & 12.97 & 6050 & 35.70 & 45.72 \\
\hline 193 & 7.35 & 15.44 & 6130 & 33.13 & 44.08 \\
\hline 194 & 6.24 & 16.20 & 5820 & 33.21 & 44.34 \\
\hline 195 & 5.83 & 13.46 & 6230 & 35.00 & 45.71 \\
\hline 196 & 5.00 & 12.97 & 6270 & 35.13 & 46.89 \\
\hline 197 & 5.18 & 15.34 & 6060 & 34.48 & 45.01 \\
\hline 198 & 5.14 & 13.40 & 6150 & 35.81 & 45.65 \\
\hline 199 & 6.10 & 11.96 & 6280 & 35.80 & 46.14 \\
\hline 200 & 5.33 & 13.26 & 6210 & 34.89 & 46.52 \\
\hline 201 & 5.45 & 13.35 & 6260 & 34.09 & 47.10 \\
\hline 202 & 5.52 & 13.36 & 6250 & 34.42 & 46.70 \\
\hline
\end{tabular}

RESULTS AND DISCUSSION

Data have compiled an interval of 10 points to draw the graphical representation and correlation of Gross calorific value $(\mathrm{GCV})$ with parameters of proximate analysis of coal.

Gross calorific value is one of the important parameters as per the requirements of IS 1350 (part-2) for $\mathrm{coal}^{2}$ samples to determine the quality. The above (proposed) correlation studies are based on the data of thermogravimetric analysis ${ }^{1}$ (TGA) and bomb calorimeter. ${ }^{2}$ Already correlation studies were done based on the experimental data of coal to calculate the approximate calorific value by formula and Artificial 
RASĀYAN J. Chem.

Vol. 13 | No. 4 |2515-2522| October - December | 2020

Neural Network (ANN) models. ${ }^{4-6}$ The proposed correlation studies are showing that the content of gross calorific value $(\mathrm{GCV})$ is increased with the decrease of the content of moisture and ash respectively (Figs.-1 and 2). The content of ash of coal is an impurity that will not be burned. The gross calorific value (GCV) is also increased with the increase of the content of volatile matters and fixed carbon respectively (Figs.-3 and 4). Volatile matters are hydrocarbons such as methane and other gases etc., Fixed carbon is carbon in the free state that does not combine with other elements. This study of gross calorific value correlated with the parameters of proximate analysis is useful for determination of the quality of coal and also it may be useful for cement industries, thermal power plants, steel making industries, etc.

\section{Moisture VS Gross Calorific Value (GCV)}

The Gross calorific value was decreased with the increase in moisture content.

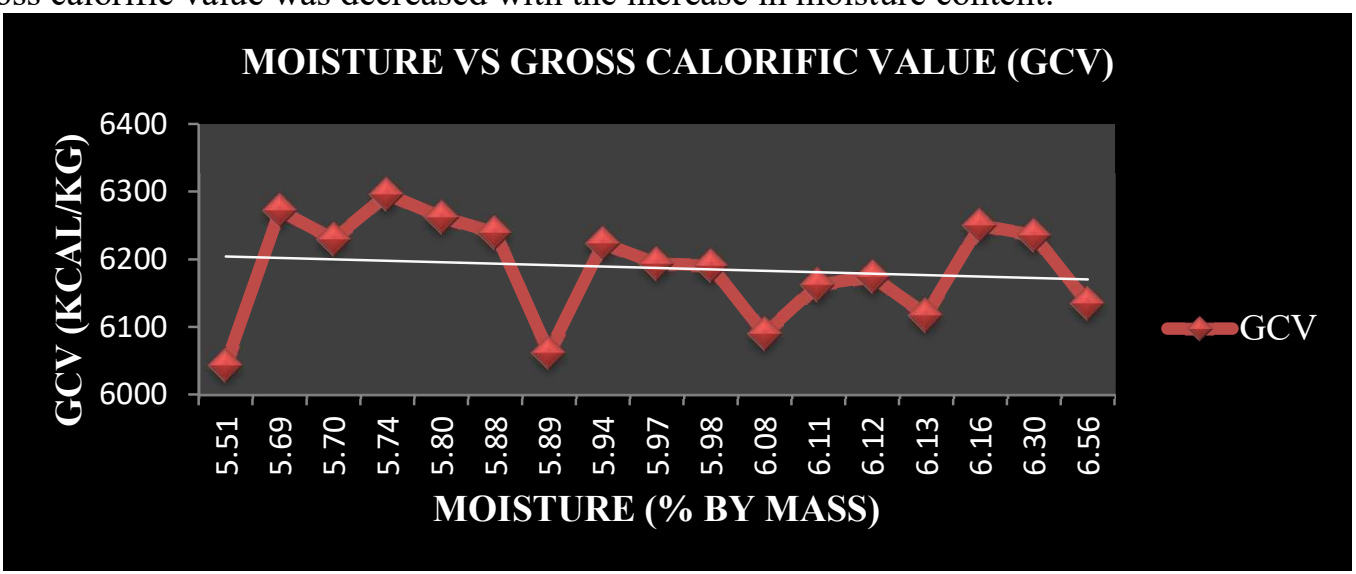

Fig.-1: Graph between Moisture VS Gross Calorific Value (GCV)

Moisture always lowers the heating value of coal, when moisture increases automatically gross calorific value decreases.

\section{Ash VS Gross Calorific Value (GCV)}

The Gross calorific value was increased with the decrease of ash content.

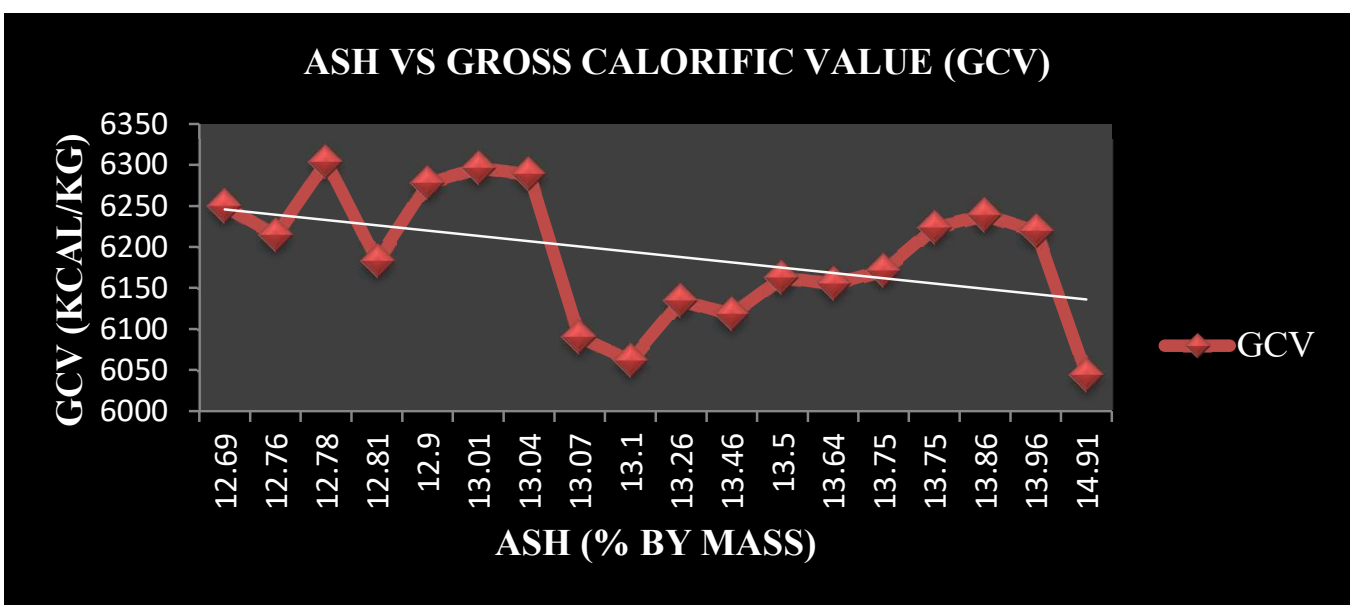

Fig.-2: Graph between Ash VS Gross Calorific Value (GCV)

Ash is an impurity will not burn. It is usually consists of silica, alumina, iron oxide, lime and magnesia etc., when high ash content, gross calorific value always decreases.

\section{Volatile Matter VS Gross Calorific Value (GCV)}

The Gross calorific value was increased with the increase of volatile matters. It consists of a complex mixture of gaseous and liquid products from thermal composition of coal. Volatile matter increases, gross calorific value also increases. 
RASĀYAN J. Chem.

Vol. 13 | No. 4 |2515-2522| October - December | 2020

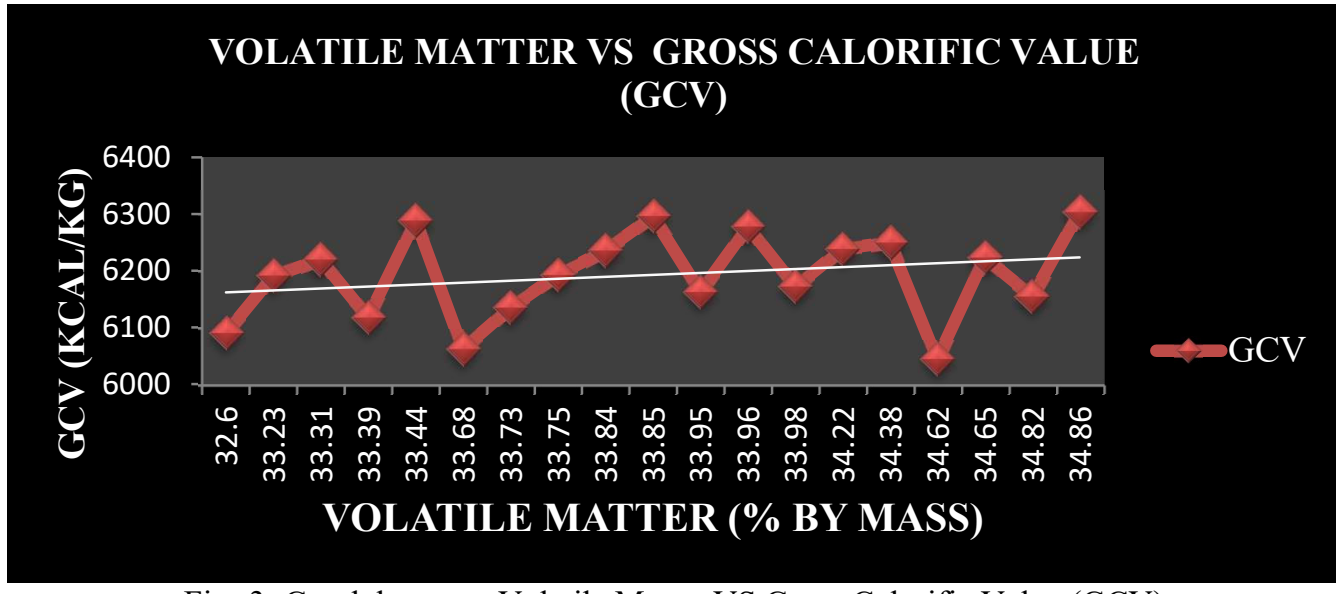

Fig.-3: Graph between Volatile Matter VS Gross Calorific Value (GCV)

\section{Fixed Carbon VS Gross Calorific Value (GCV)}

The Gross calorific value was increased with the increase of fixed carbon.

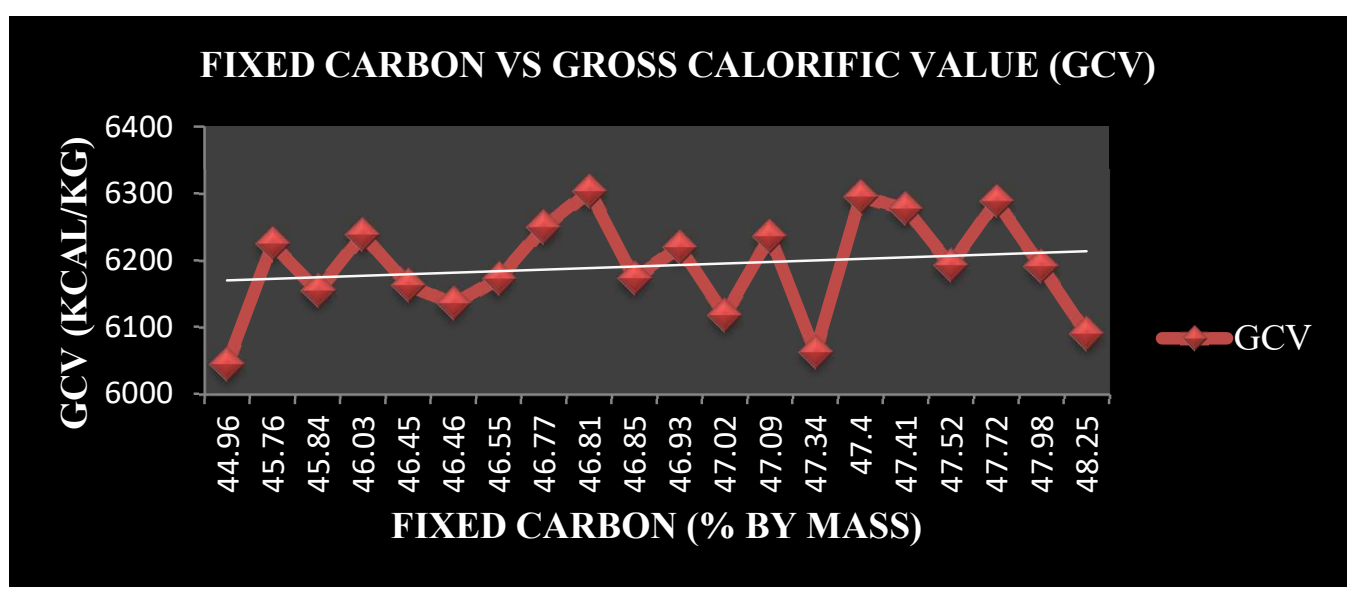

Fig.-4: Graph between Fixed Carbon VS Gross Calorific Value (GCV)

Fixed carbon calculated from 100 - (moisture + ash + volatile matter) etc., Fixed carbon is free state does not combine with any other elements. Gross calorific value increases with the increase of the high fixed carbon content.

\section{ACKNOWLEDGEMENT}

We are grateful to Sh. RakeshSaini, Scientist in-charge, NTH (NWR) Jaipur for his continuous support, motivation, and inspiration to complete the Correlation study.

\section{REFERENCES}

1. Indian Standard, Methods of Test for Coal and Coke-Part I: Proximate Analysis, IS-1350 (Part1),1984 (Reaffirmed 2019).

2. Indian Standard, Methods of Test for Coal and Coke - Determination of Gross Calorific Value by the Bomb Calorimetric Method and Calculation of Net Calorific Value (Second Revision), IS-1350(Part2),2017.

3. https://www.worldatlas.com/articles/the-top-10-coal-producers-worldwide.html.

4. Kailash Seervi, Thesis Department of Mining Engineering, National Institute of Technology Rourkela (2015).

5. Miftahul Huda, Indonesian Mining Journal, 17(1), 10(2014).

6. Mustafa Acikkar, Osman Sivrikaya, Turkish Journal of Electrical Engineering and Computer Sciences, DOI: 10.3906/elk-1802-50

[RJC-5842/2020] 